\title{
Uma Proposta de um Modelo de Decisão com Foco na Imersão para Serious Games Baseados em Vídeos 360
}

\author{
Zildomar Carlos Felix ${ }^{1,2,3}$, Liliane S. Machado ${ }^{1,2}$, Rodrigo Pinheiro de Toledo \\ Vianna $^{2}$, Júlio Raphael O. Silva ${ }^{1}$ \\ ${ }^{1}$ Universidade Federal da Paraíba -UFPB - LabTEVE, Brasil \\ ${ }^{2}$ Programa de Pós-Graduação em Modelos de Decisão e Saúde-UFPB, Brasil \\ ${ }^{3}$ Universidade Federal Rural de Pernambuco - UAST/UFRPE, Brasil \\ zildomar.felix@ufrpe.br, lilianeddi.ufpb.br, vianna@ccs.ufpb.br, \\ jraphaeldeng.ci.ufpb.br
}

\begin{abstract}
The use of $360^{\circ}$ videos has presented itself as a promising medium to promote immersion in Serious Games (SG). However, challenges remain, one of the main ones being ensuring that players do not lose focus on the narrative elements. Thus, the present study presents a proposal for a decision model focused on immersion that uses techniques to direct and attract attention to such elements. The model is being implemented in a $S G$ to raise awareness of domestic violence against women.
\end{abstract}

Resumo. $O$ uso de vídeos $360^{\circ}$ têm se apresentado como uma mídia promissora para promover imersão em Serious Games (SG). No entanto, ainda existem desafios, um dos principais é garantir que os jogadores não percam o foco nos elementos narrativos. Assim, o presente estudo apresenta uma proposta de um modelo de decisão com foco na imersão que usa técnicas para direcionar e atrair a atenção em tais elementos. $O$ modelo está sendo implementado em um $S G$ para a conscientização em violência doméstica contra a mulher.

\section{Introdução}

A violência doméstica contra a mulher vem sendo nos últimos anos um dos problemas sociais e de saúde pública mais graves enfrentados pela sociedade brasileira, e como tal, precisa ser enfrentado (PIEROTTI et al., 2018). Assim, é importante desenvolver ações que possam contribuir para o combate e enfrentamento da Violência Doméstica Contra a Mulher (VDCM). As tecnologias de informação e comunicação podem ser um fio condutor de muitas dessas ações, atuando principalmente na educação em saúde como meio transformador do conhecimento. Neste contexto, as tecnologias com propósitos educacionais, da qual os Serious Games (SG) fazem parte, vem se apresentando como um recurso promissor em diversos setores da sociedade, inclusive na educação em saúde, setor essencial na conscientização do cidadão.

Embora o tema apresente grande relevância tanto do ponto de vista social quanto de saúde pública, ainda existe uma escassez de SG que abordem essa temática. Contudo, é necessário avançar não apenas na direção de produzir jogos que colaborem no enfrentamento da VDCM como também que promovam engajamento dos jogadores de modo a maximizar as possibilidades de aprendizado e conscientização sobre os conceitos e as situações que envolvem à VDCM. 
Do ponto de vista do engajamento, novas mídias e dispositivos de Realidade Virtual (RV) vem impulsionando o desenvolvimento de ambientes cada vez mais imersivos, propiciando melhores chances de engajamento em tais ambientes. Uma tecnologia promissora para experimentar RV é o uso de vídeos $360^{\circ}$ (CHOI et al., 2018). Segundo ARGYRIOU et al. (2016), eles oferecem experiências mais realistas e aprimoradas que, quando combinadas às técnicas de jogos, tem o potencial de gerar experiências imersivas e envolventes. Diante deste cenário, o presente estudo tem a finalidade de propor um modelo de decisão que vise potencializar o senso de imersão em um SG que utiliza vídeos $360^{\circ}$ como ambiente narrativo para conscientização quanto à VDCM. Os vídeos $360^{\circ}$ utilizam um conceito visual envolvente (HAAKE, 2019), porém estão acompanhados de desafios, principalmente no que se refere ao papel do jogador (TONG et al., 2019).

\section{Serious Games e Violência Doméstica Contra a mulher}

De acordo com Dörner et. al (2016), um SG é um jogo digital que foi desenvolvido com a intenção de entreter e atingir pelo menos um objetivo adicional. Conceitualmente os SGs buscam atingir um propósito além da diversão e entretenimento. No entanto, isso não significa que eles não devam ser divertidos (DÖRNER et al., 2016), contudo, é necessário que haja um equilíbrio entre os aspectos lúdicos e educacionais. De forma complementar, Carvalho (2017) alerta que esse equilíbrio não é uma tarefa trivial, sendo, que o primeiro passo é pensar quais são os pontos fundamentais no processo de design do jogo, independentemente de ser um SG. No que tange aos serious games aplicados à VDCM, ainda existe uma grande escassez segundo Felix et al. (2018).

A VDCM é uma grave violação dos direitos humanos sofrida pelas mulheres e, como tal, precisa ser enfrentada de forma ampla com ações que possam alcançá-la seja qual for a camada social. É preciso pensar em melhorias não apenas nos efeitos da violência, mas na ampliação da promoção a saúde e prevenção da violência, ou seja, ações que possam concorrer positivamente à sua eliminação. Assim, é importante pensar em estratégias que possam levar esse conhecimento além das fronteiras dos serviços públicos de saúde. Neste contexto, os SGs se apresentam como uma abordagem inovadora, podendo ser usados para transmitir um conteúdo pedagógico estimulando e encorajando os jogadores no desenvolvimento de suas habilidades e conhecimento sobre a temática da VDCM em qualquer tempo e em qualquer lugar.

\section{Proposta de um modelo de decisão imersivo}

A proposta apresentada neste trabalho faz parte do desenvolvimento de um Serious Game de conscientização no tocante à VDCM. O jogo utiliza o formato de vídeos $360^{\circ}$ para trabalhar sua narrativa. Em particular, a proposta do modelo de decisão visa potencializar a imersão do jogador em vídeos $360^{\circ}$.

Uma grande preocupação na utilização dos vídeos $360^{\circ} \mathrm{em} \mathrm{SG}$, é garantir que o espectador não perca elementos narrativos importantes porque está olhando na direção errada (ARGYRIOU et al., 2016). Uma maneira de minimizar esse problema é criar mecanismos que possam tanto atrair a atenção do jogador quanto direcioná-lo para os elementos narrativos. Existem várias técnicas que podem ser trabalhadas nesse processo. Algumas utilizadas durante a gravação dos vídeos $360^{\circ}$, e outras na produção das cenas que serão integradas no jogo a partir de game engines. A Tabela 1 apresenta diversas dessas técnicas. 
IX Congresso Brasileiro de Informática na Educação (CBIE 2020)

Anais Estendidos do XXXI Simpósio Brasileiro de Informática na Educação (SBIE 2020)

Tabela 1: Técnicas de exploração/Direcionamento

\begin{tabular}{l|l|l|l}
\hline Mecânicas & Categoria & Uso/Fase & Referência \\
\hline \multirow{4}{*}{\begin{tabular}{c} 
Direcionar e $\begin{array}{c}\text { atrair a } \\
\text { atenção }\end{array}$ \\
\cline { 2 - 4 }
\end{tabular}} & Movimentos & Gravação & Sheikh (2016); Keskinen et al. (2019). \\
\cline { 2 - 4 } & Elementos gráficos & $\begin{array}{l}\text { Gravação; } \\
\text { Cena do Jogo. }\end{array}$ & Argyriou et al. (2016). \\
\cline { 2 - 4 } & Dicas sociais & Gravação & Sheikh et al. (2016); Ton (2019). \\
\cline { 2 - 4 } & Marcadores visuais & Cena do jogo & Argyriou et al. (2016). \\
\cline { 2 - 4 } & Conteúdos pop-up & Cena do jogo & Kallioniemi et al. (2018); \\
\hline \multirow{2}{*}{\begin{tabular}{l} 
Informacional (2016); \\
\hline
\end{tabular}} & Mini-mapas & Cena do jogo & Argyriou et al. (2016). \\
\hline
\end{tabular}

O modelo de decisão proposto neste estudo procura não apenas implementar esses mecanismos como também usar estratégias de reforço positivo (feedback) para maximizar o engajamento do jogador. $\mathrm{O}$ objetivo é estimular o jogador para que ele possa se sentir parte da experiência como um todo, englobando toda a sua atenção. A Figura 1 apresenta a arquitetura do modelo proposto.

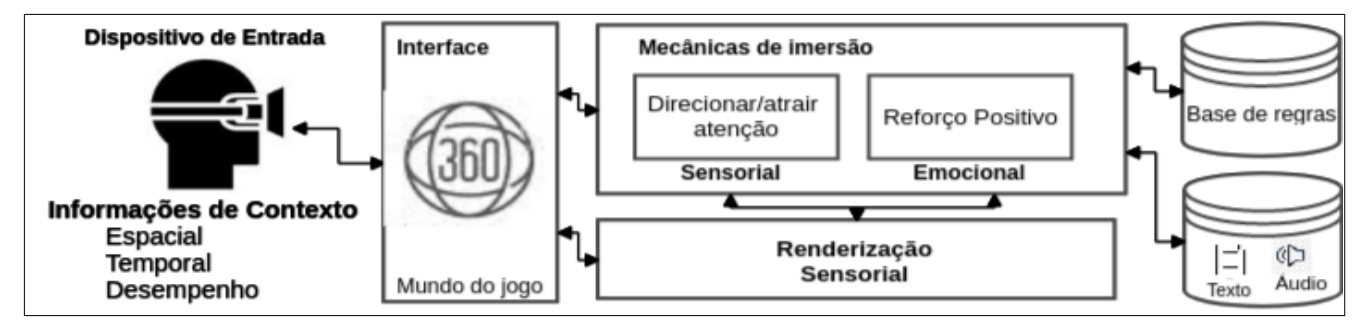

Figura 1: Arquitetura do modelo de decisão

De uma forma simplificada, pode-se dizer que o modelo percebe as informações do jogador a partir do dispositivo de entrada do ambiente (Gaze) e dos dados de imersão (dados espaciais e temporais) e de desempenho (número de desafios, taxa de desempenho). Os dados percebidos são processados pelas mecânicas de imersão a partir de um conjunto de regras e o ambiente $360^{\circ}$ é renderizado sensorialmente. A renderização sensorial no jogo consiste na ideia de potencializar a experiência do jogador com a adição de efeitos visuais e sonoros, podendo aumentar sua imersão sensorial e potencialmente a emocional ao conteúdo narrativo.

Quando imerso no ambiente virtual, o usuário (jogador) têm a sensação de fazer parte deste ambiente passando um sentimento de pleno envolvimento com o ambiente (SHERMAN; CRAIG, 2018). Neste caso, o envolvimento está sendo tratado no modelo de imersão sob duas perspectivas, a imersão sensorial e a imersão emocional. A imersão sensorial está sendo tratada sob o ponto de vista da RV, ou seja, na execução de recursos audiovisuais que permite que o jogador possa ficar focado no mundo do jogo e nos seus estímulos, possibilitando maior envolvimento e engajamento. Tais recursos são: elementos gráficos direcionais, sistemas de sons ajustáveis e direcionais, mini-mapas com posição espacial de elementos narrativos (p. ex. desafios não explorados).

Já imersão emocional, está relacionada aqui como elemento da satisfação, isto é, o grau de motivação ou satisfação que o jogador sente em realizar as atividades no mundo 
do jogo. Essa abordagem vai ao encontro a teoria da autodeterminação (SDT) proposta por Ryan e Deci (2000). A SDT aborda principalmente fatores que permite a motivação intrínseca. O sentimento de satisfação pode envolver emocionalmente o jogador na "estória" do jogo, proporcionando experiências mais ricas e prazerosas (TAMBORINI, 2010). O modelo de decisão faz uso de estratégias pedagógicas de reforço positivo para motivar o jogador a superar os desafios do jogo. A principal estratégia é o feedback de mensagens textuais e audiovisuais que são renderizadas ao longo do jogo.

O modelo detecta (ou percebe) padrões no processo de interação. Tais padrões incluem exploração do ambiente, desafios e tarefas. Por fim, a tomada de decisão é realizada na forma de ações orientadas ao conhecimento preestabelecido na base de conhecimento. A Tabela 2 exemplifica tipos de mecânicas e alguns dados de entrada que são percebidos de forma automática pelo modelo. Já as Tabelas 3 e 4 exemplificam algumas situações do jogo VDCM no qual o modelo avalia e decide no intuito de aumentar a imersão do jogador.

Tabela 2: Dados de Entrada vs Mecânica do Modelo

\begin{tabular}{l|l|l}
\hline Dado de entrada & Informação & Mecânica \\
\hline Posição espacial do jogador & Espacial & Sensorial \\
\hline Tempo de permanência em uma zona de interesse (ZI) & Temporal & Sensorial \\
\hline Número de desafios concluídos & Desempenho & Emocional \\
\hline Taxa de desempenho individual (Pontuação) & Desempenho & Emocional \\
\hline
\end{tabular}

Tabela 3: Reforço Positivo - Engajar o jogador no conteúdo apresentado

\begin{tabular}{l|l|l|l|l}
\hline Ação & Quando avaliar & Percepção & Quando Executa & Técnica \\
\hline $\begin{array}{l}\text { Motivar o jogador } \\
\text { em relação ao seu } \\
\text { desempenho; }\end{array}$ & $\begin{array}{l}\text { Desempenho nos } \\
\text { desafios do jogo; }\end{array}$ & $\begin{array}{l}\text { Desempenho } \\
\text { individual; Nível; } \\
\mathrm{N}^{\circ} \text { de desafios. }\end{array}$ & $\begin{array}{l}\text { Antes do último } \\
\text { desafio; }\end{array}$ & $\begin{array}{l}\text { Alerta textual } \\
\text { ou sonoro - } \\
\text { Voz }\end{array}$ \\
\hline $\begin{array}{l}\text { Alertar o jogador } \\
\text { sobre a necessidade } \\
\text { de lê os desafios; }\end{array}$ & $\begin{array}{l}\text { Tempo insuficiente } \\
\text { para visualização e } \\
\text { leitura da pergunta } \\
\text { do desafio. }\end{array}$ & $\begin{array}{l}\text { Tempo gasto na } \\
\text { visualização dos } \\
\text { desafios; desafios } \\
\text { visualizados; }\end{array}$ & $\begin{array}{l}\text { A partir do segundo } \\
\text { desafio visualizado; }\end{array}$ & $\begin{array}{l}\text { Alerta textual } \\
\text { ou sonoro - } \\
\text { Voz }\end{array}$ \\
\hline $\begin{array}{l}\text { Alertar o jogador } \\
\text { sobre a necessidade } \\
\text { de lê as reflexões; }\end{array}$ & $\begin{array}{l}\text { Tempo insuficiente } \\
\text { para a leitura da } \\
\text { reflexão do desafio. }\end{array}$ & $\begin{array}{l}\text { Tempo gasto na } \\
\text { visualização dos desafios; } \\
\text { desartir do segundo }\end{array}$ & $\begin{array}{l}\text { A parterta textual } \\
\text { desafio visualizado; } \\
\text { ou sonoro - } \\
\text { Voz }\end{array}$ \\
\hline
\end{tabular}

Tabela 4: Atrair e direcionar a atenção - envolvimento nos desafios

\begin{tabular}{l|l|l|l|l}
\hline Ação & Quando avaliar & Percepção & Quando Executa & Técnica \\
\hline $\begin{array}{l}\text { Alerta visual } \\
\text { sobre desafios } \\
\text { não explorados. }\end{array}$ & $\begin{array}{l}\text { Quando o jogador } \\
\text { não explora os } \\
\text { desafios. }\end{array}$ & $\begin{array}{l}\text { Desafios } \\
\text { completados; } \\
\text { Tempo na ZIs; }\end{array}$ & $\begin{array}{l}\text { O jogador ultrapassa o } \\
\text { exploração dos desafios; }\end{array}$ & $\begin{array}{l}\text { Hotspots, } \\
\text { Mini- } \\
\text { mapas. }\end{array}$ \\
\hline $\begin{array}{l}\text { Ajuste no volume } \\
\text { do áudio }\end{array}$ & $\begin{array}{l}\text { Movimentação do } \\
\text { jogador (ZIs) }\end{array}$ & $\begin{array}{l}\text { Posição espacial } \\
\text { do jogador }\end{array}$ & $\begin{array}{l}\text { Movimento da cabeça do } \\
\text { jogador. }\end{array}$ & $\begin{array}{l}\text { Audio } \\
\text { espacial }\end{array}$ \\
\hline $\begin{array}{l}\text { Sugerir repetição } \\
\text { dos desafios. }\end{array}$ & $\begin{array}{l}\text { O jogador não } \\
\text { permaneceu na ZI } \\
\text { o tempo mínimo }\end{array}$ & $\begin{array}{l}\text { Visualizou ZI } \\
\text { (2x); Tempo de } \\
\text { permanência ZI. }\end{array}$ & $\begin{array}{l}\text { Quando o jogador passar } \\
\text { pela segunda vez pela ZI }\end{array}$ & $\begin{array}{l}\text { Elementos } \\
\text { gráficos }\end{array}$ \\
\hline
\end{tabular}

As abordagens utilizadas na concepção do modelo de decisão, foram pensadas nas particularidades do emprego dos vídeos $360^{\circ}$ em SG. A preocupação vai desde da gravação das cenas até integração com o jogo. Isso se faz necessário porque parte do direcionamento e atração da atenção do jogador poderia ser implementadas durante a gravação dos vídeos $360^{\circ}$, isso pode facilitar o envolvimento do jogador de forma natural, sem a necessidade de muitas intervenções por parte do modelo de decisão. O excesso de intervenções poderia causar o efeito inverso, ou seja, desengajar ou até quebrar a imersão. 


\section{Considerações Finais}

O uso de vídeos $360^{\circ}$ em SG quando visualizados em dispositivos especiais como os óculos de RV para dispositivos móveis, oferecem novas possibilidades de experiências imersivas (HAAKE, 2019). Também apresenta novos desafios. Uma questão significativa está associada à perda de foco dos jogadores. Foi apresentada aqui uma proposta de um modelo de decisão que integra diversas destas técnicas com o objetivo de engajar e estimular os jogadores através de experiências mais imersivas, garantindo que os elementos importantes da narrativa do SG sejam explorados a partir de mecânicas de imersão sensorial e emocional. Este modelo está sendo empregado no design de um SG em conscientização em relação à VDCM no sentido de enriquecer a experiência de seus jogadores e, sobretudo, no enfrentamento desta importante temática.

\section{Referências}

Argyriou, L., Economou, D., Bouki, V., \& Doumanis, I.. Engaging immersive video consumers: Challenges regarding 360-degree gamified video applications. In:15th International Conference on Ubiquitous Computing. 2016. p. 145-152.

Choi, K., Yoon, Y. J., Song, O. Y., \& Choi, S. M. Interactive and immersive learning using $360^{\circ}$ virtual reality contents on mobile platforms. Mobile Information Systems, 2018.

Dörner, R., Göbel, S., Effelsberg, W., \& Wiemeyer, J. . Serious games: foundations, concepts and practice. Springer, 2016.

Felix, Z. C., Machado, L. S., Silva, J. R. O., Silva, A. T. M. C., Almeida, L. R. Avaliando Satisfação do Usuário a Partir dos Modelos GameFlow e PENS: Um Estudo com o Jogo Caixa de Pandora Mobile. Proceedings of SBGames, 2018.

Haake, Susanne; Müller, Wolfgang. Cyberella-Design Issues for Interactive 360 Degree Film. In: Interactivity, Game Creation, Design, Learning, and Innovation. 2018.

Keskinen, T., et al. The Effect of Camera Height, Actor Behavior, and Viewer Position on the User Experience of 360 Videos. In: 2019 IEEE Conference on Virtual Reality and 3D User Interfaces (VR). IEEE, 2019. p. 423-430.

Pierotti, C. F.; D’Oliveira, A. F. P. L.; Terra, M. F. A situação de violência doméstica de gênero na atenção primária à saúde. Arquivos Médicos dos Hospitais e da Faculdade de Ciências Médicas da Santa Casa de São Paulo, p. 12-18, 2018.

Ryan, Richard M.; Deci, Edward L. Self-determination theory and the facilitation of intrinsic motivation, social development, and well-being. American psychologist, v. 55, n. 1, p. 68, 2000.

Sheikh, Alia et al. Directing attention in 360-degree video. 2016.

Sherman, w. R.; Craig, A. B. Understanding virtual reality: Interface, application, and design. 2. ed. Morgan Kaufmann, 2018.

Tamborini, Ron et al. Defining media enjoyment as the satisfaction of intrinsic needs. Journal of communication, v. 60, n. 4, p. 758-777, 2010.

Tong, lingwei; jung, sungchul; lindeman, robert w. Action Units: Directing User Attention in 360-degree Video based VR. In: 25th ACM Symposium on Virtual Reality Software and Technology. 2019. 